\title{
AFINIDADE OU ASPIRAÇÃO? GRUPOS DE REFERÊNCIA VALORIZADOS POR CONSUMIDORAS DE BAIXA RENDA ${ }^{1}$
}

\author{
Carolina Araujo Forleo ${ }^{2}$ \\ Luiz Antonio Slongo ${ }^{3}$
}

http://dx.doi.org/10.1590/1413-2311.249.91706

\section{RESUMO}

Considerando-se a importância das relações sociais e da influência interpessoal no comportamento dos indivíduos de baixa renda, este estudo objetiva identificar e analisar os grupos de referência valorizados pelas consumidoras desse segmento em seu processo de decisão de compra. A coleta de dados fundamenta-se em uma abordagem multimétodo composta, primeiro, por entrevistas em profundidade com 12 mulheres e, depois, pela técnica de Análise Conjunta com uma amostra de 53 entrevistadas pertencentes a esse segmento. Devido à crise econômica vivida durante o período de coleta de dados, o contexto da pesquisa é delimitado a um bem que permanece atrativo nessas circunstâncias: o batom. Os resultados obtidos demonstram que as consumidoras de baixa renda valorizam a presença de diferentes grupos de referência em seu processo de decisão. Especificamente, identificam-se dois grupos de respondentes com preferências distintas quanto à participação aos seguintes grupos de referência: familiares, amigas, consultoras de venda, vizinhas/colegas, celebridades, blogueiras e mulheres com melhores condições financeiras.

Palavras-chaves: Comportamento do Consumidor. Consumidores de Baixa Renda. Grupos de Referência.

\footnotetext{
${ }^{1}$ Submetido em 10/04/2019; aprovado em 29/07/2019.

${ }^{2}$ Pesquisadora independente; Porto Alegre - RS (Brasil) - http://orcid.org/000-0001-9465-5793. E-mail: forleo.carolina@gmail.com.

${ }^{3}$ Universidade Federal do Rio Grande do Sul (UFRGS) - Escola de Administração e PPGA; Porto Alegre - RS (Brasil) - http://orcid.org/0000-0002-8269-4338. E-mail: luiz.slongo@ufrgs.br.
} 


\title{
AFFINITY OR ASPIRATION? REFERENCE GROUPS VALUED BY LOW- INCOME FEMALE CONSUMERS
}

\begin{abstract}
Considering the importance of social relations and interpersonal influence on the behavior of low-income individuals, this study aims to identify and analyze the reference groups valued by low-income female consumers in their decision-making process. A multimethod approach was employed. First, the data were collected through in-depth interviews with 12 low-income women. Subsequently, a Conjoint Analysis technique was applied with a sample of 53 female interviewees belonging to this segment. Due to the economic crisis experienced during the period of data collection, the context of the research was limited to a good that remains attractive in these circumstances: lipstick. Results showed that low-income female consumers value the presence of different reference groups in their decision-making process. Specifically, it was possible to identify two groups of respondents with distinct preferences regarding the following reference groups: family, friends, sales consultants, neighbors/colleagues, celebrities, bloggers, and women in a better financial condition.
\end{abstract}

Keywords: Consumer behavior. Low-income consumers. Reference groups.

\section{¿AFINIDAD O ASPIRACIÓN? GRUPOS DE REFERENCIA VALORADOS POR CONSUMIDORAS DE BAJOS INGRESOS}

\begin{abstract}
RESUMEN
Teniendo en cuenta la importancia de las relaciones sociales y de la influencia interpersonal en el comportamiento de los individuos de bajos ingresos, este estudio tiene por objetivo identificar y analizar los grupos de referencia valorados por las consumidoras de bajos ingresos en su proceso de decisión de compra. La recolección de datos utiliza un enfoque multimétodo. En primer lugar, se recopilaron los datos se recopilaron a través de entrevistas en profundidad con 12 mujeres de bajos ingresos. Luego, se aplicó una técnica de Conjoint Analysis con una muestra de 53 entrevistadas pertenecientes a este segmento. Debido a la crisis económica vivida durante el período de recolección de datos, el contexto de la investigación quedó delimitado a un bien que sigue siendo atractivo en estas circunstancias:
\end{abstract}


el lápiz labial. Los resultados obtenidos demuestran que las consumidoras de bajos ingresos valoran la presencia de diferentes grupos de referencia en su proceso de decisión de compra. Específicamente, se identifican dos grupos de encuestados con preferencias distintas en lo que respecto a la participación de los siguientes grupos de referencia: familiares, amigas, representantes de venta, vecinas/colegas, celebridades, bloggers y mujeres con mejores condiciones financieras.

Palabras-clave: Comportamiento del consumidor. Consumidores de bajos ingresos. Grupos de referencia.

\section{INTRODUÇÃO}

A partir dos anos 2000, os indivíduos brasileiros de baixa renda passaram a ser reconhecidos como consumidores nos âmbitos acadêmico e mercadológico. Uma série de fatores, sobretudo o aumento do poder aquisitivo e, consequentemente, do poder de compra, estimulou o desenvolvimento de um corpo teórico sobre o comportamento desses consumidores (NOGAMI; PACAGNAN, 2011). Dentre os principais temas abordados pelos pesquisadores, destaca-se o processo de decisão de compra desses indivíduos, principalmente a identificação de atributos que os auxiliam a determinar suas escolhas (BARROS, 2006; CHAUVEL; MATTOS, 2008; LIVRAMENTO; HOR-MEYLL; MATTOS, 2007; PRAHALAD, 2005; PESSÔA, 2013).

A importância das relações sociais também é evidenciada em alguns estudos sobre o comportamento dos consumidores brasileiros de baixa renda. Observa-se, por exemplo, que esses indivíduos estão inseridos em um contexto altamente relacional, marcado pelo compartilhamento de bens e espaços, pelo senso de comunidade e pela rede de sociabilidade (BARROS, 2006; CASTILHOS; ROSSI, 2009). Além disso, entende-se que o consumo desse público associa-se à necessidade de inclusão e também de distinção social (BARBOSA; HOR-MEYLL; MOTTA, 2009; CHAUVEL; MATTOS, 2007).

Apesar desse avanço na investigação sobre a influência social no comportamento desse segmento, constata-se pouco avanço no que diz respeito à compreensão dos grupos de referência que participam de seu processo de decisão de compra. Ao abordar outros assuntos relativos ao comportamento desses indivíduos, alguns pesquisadores identificaram os familiares, os vizinhos e grupos de classe social superior como grupos de referência que 
influenciam esse público (BARBOSA; HOR-MEYLL; MOTTA, 2009; BARROS, 2006; VASCONCELOS; GOMES; LEOCÁDIO, 2015). Diante disso, identifica-se que há espaço para o desenvolvimento de novos estudos que contemplem essa temática, pois não existem evidências sobre a influência de outros grupos, como celebridades e especialistas, nesse processo, bem como não se tem conhecimento sobre a preferência desses consumidores em relação aos diferentes grupos de referência.

Assim, o presente artigo contribui para o entendimento desse fenômeno ao identificar e analisar os grupos de referência valorizados por consumidoras de baixa renda em seu processo de decisão de compra. Especificamente, este estudo objetiva identificar e analisar os grupos de afinidade e de aspiração valorizados por essas consumidoras, bem como verificar a existência de possíveis agrupamentos (clusters) com base na preferência por grupos de referência.

Entende-se que a compreensão dos grupos que influenciam e que são valorizados por esse segmento mostra-se importante para a academia e para o mercado. Por um lado, isso significa adquirir conhecimento mais completo sobre o processo de decisão de compra desse público. Por outro lado, esse entendimento possibilita apontar sugestões e direcionamentos sobre quais grupos de referência podem ser utilizados por empresas que atuam junto a esse segmento.

\section{REFERENCIAL TEÓRICO}

A fim de aprofundar este estudo, o referencial teórico aborda temáticas relacionadas ao processo de decisão de compra e influência social, aos grupos de referência e ao consumidor de baixa renda. Ressalta-se que para os dois primeiros tópicos foram considerados estudos nacionais e internacionais, pois se entende que estes são temas universais na literatura de comportamento do consumidor. No entanto, para o terceiro tópico foram priorizadas pesquisas realizadas no contexto brasileiro, uma vez que os consumidores pobres do Brasil apresentam características, hábitos e padrões específicos que os diferem de indivíduos provenientes de outros países (PRAHALAD; LIEBERTHAL, 2003).

\subsection{PROCESSO DE DECISÃO DE COMPRA E INFLUÊNCIA SOCIAL}

As pessoas são continuamente expostas a escolhas de produtos, serviços e marcas. Por isso, o processo de decisão de compra é um elemento central na teoria de comportamento do 
consumidor. Em 1968, Engel, Kollatt e Blackwell propuseram um modelo de tomada de decisão que, após revisões, alterações e correções, permanece como uma pedra angular na disciplina de marketing. Trata-se de um processo sequencial constituído por cinco etapas: reconhecimento do problema, busca de informações, avaliação de alternativas, compra, e avaliação pós-consumo (ASHMAN; SOLOMON; WOLNY, 2015).

Dado o atual contexto de cultura participativa digital e socialização on-line, algumas fases, como a avaliação de alternativas, tornaram-se mais eficientes e rápidas, enquanto outras etapas, como a avaliação pós-compra, estão mais prolongadas e complexas (ASHMAN; SOLOMON; WOLNY, 2015). No cenário contemporâneo, revela-se ainda o comportamento em canais cruzados, isto é, a utilização simultânea dos canais on-line e offline em uma tomada de decisão. Ao adotar esse tipo de comportamento, os consumidores conseguem tornar o processo de compra mais eficiente, obter ganhos e evitar custos ou riscos (PORTO; OKADA, 2018).

Independente dos canais utilizados, observa-se que a decisão de compra é moldada e influenciada por diferentes aspectos internos e externos. Uma maneira de compreender essas influências é a categorização em quatro tipos de fatores: culturais, sociais, pessoais e psicológicos (MEDEIROS; CRUZ, 2006). Do ponto de vista social, considera-se que os consumidores utilizam sugestões e opiniões de outras pessoas como fontes de informação para moldar a sua experiência com um bem ou marca, para avaliar produtos e para fazer suas decisões finais de compra (ASHMAN; SOLOMON, WOLNY, 2015; ESCALAS; BETTMAN, 2003; RILEY; GOSLINE, 2011).

A influência interpessoal é descrita a partir de duas dimensões. A primeira é a influência informacional que ocorre quando o indivíduo busca informações confiáveis junto a outras pessoas que tenham conhecimento e experiência com um produto ou marca. A segunda é a influência normativa que se origina do desejo de alcançar pertencimento, identificação ou aprovação social. Trata-se da vontade de ser aceito e de estar em conformidade com as expectativas, padrões e normas do grupo. Costuma-se subdividir essa influência em dois tipos: utilitária e expressiva de valor. A utilitária refere-se às tentativas de cumprir os desejos de outros a fim de receber recompensas ou evitar punições. Já, a expressiva de valor caracteriza-se por ser um processo de identificação através do qual as pessoas tentam melhorar sua imagem associando-se a grupos avaliados positivamente e distanciando-se daqueles julgados negativamente (BEARDEN; NETEMEYER; TEEL, 1989; LEAL; HORMEYLL; PESSÔA, 2014; PARK; LESSIG, 1977). 
De forma semelhante, Bragaglia e Bastos (2017) destacam que o consumo é um fenômeno central na sociedade contemporânea e, por conseguinte, a aquisição de bens e marcas é utilizada como sinal de reconhecimento e relacionamento entre as pessoas ou grupos de pessoas. Nessa perspectiva, o indivíduo utiliza produtos e marcas para expor sua identidade, e também para expressar uma boa imagem com a intenção de obter aprovação social e a sensação de pertencimento a determinado grupo. Isso evidencia o significado social intrínseco ao consumo e demonstra que o processo de decisão de compra pode ser considerado um processo social (GUILLEN-ROYO, 2011; RILEY; GOSLINE, 2011), em que é notória a relevância de agrupamentos, em especial dos grupos de referência.

\subsection{GRUPOS DE REFERÊNCIA}

A expressão 'grupos de referência' é utilizada para descrever qualquer pessoa ou grupo - real ou imaginário - que influencia de modo significativo as avaliações, as aspirações e o comportamento de um indivíduo (BEARDEN; ETZEL, 1982; PARK; LESSIG, 1977). Frequentemente, os consumidores utilizam grupos de referência para guiar suas decisões de compra, seja perguntando a seus amigos e familiares sobre um produto ou marca antes de tomarem uma decisão, seja fundamentando as suas compras no endosso de alguma celebridade ou outro influenciador (HOONSOPON; PURIWAT, 2016).

As referências utilizadas podem ser socialmente próximas, com influência restrita ao ambiente imediato do consumidor e com quantidade significativa de interação direta (CHILDERS; RAO, 1992). Esses são os grupos privados - por exemplo, familiares e amigos - que demonstram ter maior impacto na intenção de compra (HOONSOPON; PURIWAT, 2016). Além disso, as referências podem ser socialmente distantes, com impacto sobre muitas pessoas e relativamente pouca ou nenhuma interação direta, apenas a observação do comportamento (CHILDERS; RAO, 1992). Trata-se do grupo público que envolve, por exemplo, as celebridades e os especialistas (HOONSOPON; PURIWAT, 2016; LEAL; HORMEYLL; PESSÔA, 2014).

As celebridades podem ser descritas como figuras públicas bastante conhecidas - por exemplo, atores, atletas, músicos, modelos, chefs de cozinha -, que recebem atenção significativa da mídia de massa. A eficiência da utilização desses indivíduos para influenciar o comportamento do consumidor depende da credibilidade e da atratividade da fonte (MILLER; LACZNIAK, 2011). Já os especialistas, ou experts, são indivíduos com ampla base de conhecimento, informações, experiências ou habilidades em relação a determinado 
assunto. Devido a sua expertise, esse grupo tende a demonstrar opinião considerada verossímil e confiável (LEAL; HOR-MEYLL; PESSÔA, 2014).

No ambiente virtual, os formadores de opinião são chamados de influenciadores digitais e representam pontos de referência para os seus seguidores (ALMEIDA, et al., 2018; UZUNOĞLU; KIP, 2014; ALMEIDA, et al., 2018). Dentre eles, destacam-se os blogueiros que utilizam suas páginas eletrônicas para publicar suas opiniões e pensamentos, além de links e imagens (MURADIAN et al., 2014). As publicações feitas por formadores de opinião individuais geram mais engajamento do que postagens de perfis institucionais (ALMEIDA et al., 2018).

Os diferentes grupos de referência identificados na literatura podem ser classificados levando-se em consideração o tipo de influência (positiva ou negativa) e de associação (envolvimento ou não com o grupo). Ao cruzar esses fatores identificam-se quatro tipos de grupos de referência: grupo de afinidade, grupo de negação, grupo de aspiração e grupo de prevenção (SCHIFFMAN; KANUK, 2000).

O grupo de afinidade compõe-se de pessoas conhecidas que influenciam diretamente um indivíduo. Trata-se de um grupo ao qual o indivíduo pertence, com o qual tem contato direto e frequente e, cujos valores, atitudes e padrões são aprovados por ele (ESCALAS; BETTMAN, 2003; SCHIFFMAN; KANUK, 2000). Família e grupos de pares são exemplos desse tipo de referência. Por outro lado, o grupo de negação refere-se ao conjunto de indivíduos ao qual um consumidor está associado, mas cujos valores, atitudes e comportamentos ele desaprova ou rejeita. Esse indivíduo tende a buscar a distinção do grupo (SCHIFFMAN; KANUK, 2000).

Os consumidores também podem ser influenciados por grupos aos quais não pertencem. O grupo de aspiração é composto por indivíduos com os quais eles não têm contato direto, mas admiração e atração pelas normas, valores e comportamentos. As celebridades são exemplos dessas figuras idealizadas (ESCALAS; BETTMAN, 2003; SCHIFFMAN; KANUK, 2000; ESCALAS; BETTMAN, 2003). Por fim, o grupo de prevenção refere-se ao conjunto de pessoas ao qual o consumidor não está associado, e mesmo assim tenta se distanciar (ENGLIS; SOLOMON, 1995; SCHIFFMAN; KANUK, 2000). 


\subsection{CONSUMIDORES DE BAIXA RENDA}

$\mathrm{Na}$ literatura de marketing, observa-se dificuldade na formação de um consenso quanto à definição de baixa renda (NOGAMI; PACAGNAN, 2011). Neste estudo, optou-se por seguir e atualizar a proposta do Centro de Excelência em Varejo da Fundação Getúlio Vargas (CEV-FGV), que considera os consumidores de baixa renda, no Brasil, aqueles pertencentes às classes $\mathrm{D}$ e $\mathrm{E}$, ou seja, com renda familiar mensal de até $\mathrm{R} \$ 1.115,00$ (FUNDAÇÃO GETÚLIO VARGAS, 2011). Trazendo essa definição para os valores do salário mínimo em 2016, quando a pesquisa foi aplicada, considera-se baixa renda os indivíduos com renda familiar mensal de até $\mathrm{R} \$ 2.300,00$.

O processo de decisão de compra desse segmento envolve uma equação complexa de valor, em que são considerados diversos atributos e variáveis (MATTOS, 2007). Os consumidores de baixa renda preocupam-se com as suas limitações financeiras e com as capacidades do orçamento familiar. Assim, identifica-se o caráter racional na gestão de recursos e, consequentemente a atenção dada ao preço dos bens (BARROS, 2006; CHAUVEL; MATTO, 2008; MATTOS, 2007).

No entanto, nem sempre essa racionalidade predomina nas decisões, pois o público de baixa renda também expressa o desejo de pertencer à sociedade de consumo e de atender às preferências da família (BARROS, 2006; CHAUVEL; MATTO, 2008; MATTOS, 2007). Diante disso, destaca-se a importância das marcas. No processo de decisão das consumidoras de baixa renda, as marcas exercem papeis distintos. Dependendo do tipo de compra, as marcas podem servir tanto para sinalizar qualidade quanto para remeter à hierarquia social (ROCHA; SILVA, 2008). Por essa razão, as consumidoras demonstram vasto conhecimento a respeito das marcas, em diversas categorias de produtos (BARROS, 2006; LIVRAMENTO; HOR-MEYLL; PESSÔA, 2013; MATTOS, 2007).

Ao observar os fatores que influenciam o processo de decisão de compra da baixa renda, evidencia-se a presença de duas lógicas dominantes que coexistem. A primeira, proveniente da limitação financeira, estabelece uma racionalização dos gastos e atenção ao preço. A segunda, de ordem simbólica, fundamenta-se em elementos culturais, especialmente nas dimensões relacionais e hierárquicas (CHAUVEL; MATTOS, 2008). Nesse sentido, destaca-se a importância das relações pessoais e da influência social, sobretudo dos grupos de referência.

Ao explorar outras questões da experiência e dos hábitos de consumo dos consumidores de baixa renda, as pesquisas de Barbosa, Hor-meyll e Motta (2009) e 
Vasconcelos, Gomes e Leocádio (2015) evidenciam a influência de grupos de contato no comportamento desse segmento. No primeiro estudo, identifica-se a família como principal grupo de referência na aquisição de aparelhos celulares e de serviços de telefonia móvel. No segundo, observa-se que os vizinhos e os familiares são o público de maior influência no processo de compartilhamento do significado de bens e na disseminação do comportamento de consumo.

Ainda no estudo de Vasconcelos, Gomes e Leocádio (2015), é possível observar a influência dos grupos de aspiração. Os pesquisadores identificam que grupos de classe socioeconômica superior - patroas e familiares com melhores condições financeiras também participam do processo de compartilhamento do significado dos bens. De forma semelhante, Barros (2006) descreve o comportamento de empregadas domésticas que absorvem hábitos e práticas de consumo de suas patroas, embora haja releituras e ressignificações.

Esta pesquisa de Barros (2006) também identifica a existência de alguns comportamentos de distinção dos pares, sugerindo que há influência de grupos de negação ou dissociação na tomada de decisão desse público. Esse comportamento é evidenciado no estudo de Castilhos e Rossi (2009) que revela a tentativa dos indivíduos de baixa renda de, através do consumo, se diferenciar dos "pobres-pobres", aqueles que não possuem casa ou alimentos.

Com base nesse levantamento teórico, entende-se que existe espaço para o desenvolvimento de novos estudos que contemplem a temática de grupos de referência em relação ao público de baixa renda. Além disso, compreende-se a importância de pesquisar a preferência das consumidoras desse segmento tanto por grupos de afinidade quanto por grupos de aspiração.

\section{MÉTODO}

Nesta seção apresenta-se o método utilizado a fim de atender os objetivos da pesquisa. Inicialmente, abordam-se aspectos gerais da pesquisa. Em seguida, descrevem-se os procedimentos utilizados nas etapas qualitativa e quantitativa do estudo. 


\subsection{ASPECTOS GERAIS}

Para a realização deste estudo optou-se por utilizar uma abordagem multimétodo. Inicialmente, cumpriu-se uma fase de vertente qualitativa, alicerçada em entrevistas em profundidade, a fim de identificar os grupos de referência presentes no processo de decisão de consumidoras de baixa renda. Após a coleta e análise desses dados, estruturou-se e executou-se a segunda etapa, de natureza quantitativa, com a adoção da análise conjunta (conjoint analysis) como método de condução, para analisar as preferências das respondentes quanto aos grupos de referência.

Outro aspecto importante diz respeito à delimitação do contexto de pesquisa. Sabe-se que as decisões relacionadas ao consumo não são iguais em todas as ocasiões e que a influência de grupos de referência também pode mudar, dependendo da compra. Por isso, entendeu-se necessário restringir o cenário à aquisição de uma categoria de produtos. Estabeleceu-se assim que o consumo de cosméticos representa um contexto adequado, pois as mulheres de baixa renda associam-nos tanto à autoestima e à sensação de bem-estar pessoal quanto à busca por agradar ou obter o respeito de outras pessoas (LIVRAMENTO; HORMEYLL; PESSÔA, 2013). Além disso, durante períodos de crise econômica aumenta a tendência à indulgência e, consequentemente, a venda de cosméticos (CRUZ, 2016). Desse modo, entende-se que responder uma pesquisa sobre produtos de beleza não causaria estranhamento para as consumidoras, apesar da situação econômica do país durante a coleta de dados.

No entanto, essa definição permaneceu muito abrangente, mostrando-se necessário restringir ainda mais e escolher uma categoria específica dentre os produtos de beleza que fosse de alto envolvimento. Utilizou-se esse critério, pois quanto maior o nível de envolvimento, maior a disponibilidade do consumidor para vivenciar todos os estágios do processo de decisão de compra, inclusive a busca de informações em fontes externas (HOONSOPON; PURIWAT, 2016; MEDEIROS; CRUZ, 2006). Diante disso, revelou-se ser fundamental a identificação dessa categoria de produto na etapa qualitativa. Com base nas entrevistas em profundidade, observou-se a importância do batom para as consumidoras de baixa renda. Sendo assim, a compra e o uso desse produto foram usados como contexto para a fase seguinte do estudo. As análises que embasaram essa decisão são aprofundadas na seção referente aos resultados da etapa qualitativa. 


\subsection{ETAPA QUALITATIVA}

Conforme descrito, na fase qualitativa, realizaram-se entrevistas pessoais e individuais com roteiro semiestruturado, de modo que não houvesse pressão social ou influência de outras pessoas nas respostas das participantes. Ao todo foram realizadas 12 entrevistas em profundidade com mulheres entre 20 e 58 anos, residentes na Região Metropolitana de Porto Alegre. O filtro utilizado para selecionar essas respondentes foi o costume de comprar e usar produtos de beleza e a renda familiar mensal (inferior a $\mathrm{R} \$ 2.300,00$ ). O número total de entrevistas fundamenta-se na perspectiva de Gaskell (2002): uma quantidade maior de entrevistas não significa obrigatoriamente melhor qualidade ou detalhamento. Quando surgem repetições nas respostas, o pesquisador pode dedicar-se à interpretação e à análise dos dados.

Para analisar os dados obtidos na etapa qualitativa escolheu-se a técnica de análise de conteúdo, mais especificamente optou-se pela análise categorial, em que o texto dos discursos é fragmentado em categorias (BARDIN, 2011). No presente estudo, utilizaram-se as seguintes categorias a priori: (1) cosméticos de alto envolvimento; (2) influência dos grupos de referência. Ressalta-se que na análise, as entrevistadas serão identificadas pela letra E seguida de números de 1 a 12 - E1, E2...E12). Com base nos resultados dessa etapa, desenhou-se a fase quantitativa.

\subsection{ETAPA QUANTITATIVA}

A Análise Conjunta, ou conjoint analysis, é utilizada para investigar as preferências das respondentes e envolve a identificação da utilidade (valorização) associada a diferentes níveis de fatores analisados. Quanto maior a utilidade, maior a preferência. Essa estimativa é feita com base na avaliação decorrente da ordenação de diferentes estímulos. A aplicação mais frequente dessa técnica refere-se ao desenvolvimento de novos produtos e serviços, em que se analisam diferentes atributos que influenciam os consumidores (HAIR Jr. et al., 2005). No entanto, não são apenas as características dos bens que motivam a decisão de compra; relações pessoais e grupos de referência também interferem diretamente nesse processo. Além disso, os indivíduos podem valorizar de maneira diferente as fontes externas dependendo da situação de compra vivenciada. Embora o objetivo do estudo não seja o desenvolvimento de produtos e serviços, a conjoint analysis parece adequada, pois se 
pretende analisar os grupos de referência valorizados, isto é, preferidos pelas consumidoras de baixa renda.

Para utilizar essa técnica é preciso conhecer os fatores e níveis relevantes para os consumidores a fim de formular estímulos e cartões adequados. Neste estudo, esses elementos foram definidos com base nas entrevistas da etapa qualitativa (Quadro 1). Destacase que "influencia" e "não influencia" significam, respectivamente, a participação ou não dos diferentes grupos de referência no processo de decisão de compra - tanto de maneira direta (recomendação) quanto indireta (observação).

Quadro 1 - Fatores e níveis utilizados

\begin{tabular}{|l|ll|}
\hline \multicolumn{1}{|c|}{ FATORES } & \multicolumn{2}{c|}{ NíVEIS } \\
\hline Familiares & Influencia & Não influencia \\
\hline Amigas & Influencia & Não influencia \\
\hline Vizinhas/Colegas & Influencia & Não influencia \\
\hline Consultoras de venda & Influencia & Não influencia \\
\hline Celebridades & Influencia & Não influencia \\
\hline Mulheres com melhor condição financeira & Influencia & Não influencia \\
\hline Blogueiras & Influencia & Não influencia \\
\hline
\end{tabular}

Fonte: elaborado pelos autores.

Quando o número de combinações é grande torna-se cansativa e complicada a avaliação das respondentes. Por isso, os pesquisadores utilizam o método de delineamento fatorial fracionado para definir um subconjunto de estímulos (HAIR Jr. et al., 2005). No presente estudo, combinando os fatores e seus níveis, têm-se 128 estímulos. Ao gerar um design ortogonal no software de análise estatística SPSS ${ }^{\circledR}$, obteve-se um subconjunto de oito (8) estímulos. Além desses, incluíram-se mais dois (2) conjuntos com base nos resultados qualitativos. Para a exposição e mensuração desses estímulos, selecionou-se o modo de Análise Conjunta Tradicional, especificamente a exposição de perfil completo (HAIR Jr. et al., 2005), que se caracteriza pela descrição de cada estímulo em um cartão-índice separado.

Tendo em vista que se definiu o consumo de batons como contexto da conjoint analysis, considera-se que a população-alvo desta pesquisa são mulheres consumidoras de batom, pertencentes ao segmento de baixa renda - isto é, com renda familiar mensal de até $\mathrm{R} \$ 2.300,00$. Já a amostra do estudo, selecionada com base na técnica de amostragem não probabilística, compõe-se de 53 mulheres, residentes da Região Metropolitana de Porto Alegre, que costumam comprar batom pelo menos uma vez por ano e possuem renda familiar 
mensal inferior a $\mathrm{R} \$ 2.300,00$. Ao observar o estudo de outros pesquisadores, por exemplo, Prado et al. (2014), entende-se que o tamanho da amostra é adequado à técnica utilizada.

Antes da aplicação da pesquisa realizou-se um pré-teste com base em seis (6) entrevistas para avaliar o instrumento de coleta de dados, a linguagem utilizada e o método de apresentação e a mensuração dos estímulos. Finalizada a aplicação do pré-teste, desenvolveuse a etapa de coleta de dados, fundamentada em entrevistas pessoais com roteiro estruturado, o qual incluiu o ordenamento dos cartões e também as perguntas relativas ao perfil das respondentes. Na parte relativa à ordenação, os dez cartões impressos eram entregues às respondentes e solicitava-se que elas os classificassem em um tabuleiro com 10 postos do mais importante ao menos importante.

Por fim, analisaram-se os dados. Inicialmente, realizou-se a análise univariada a fim de caracterizar a amostra. Na sequência, empreendeu-se a análise multivariada, apropriada à conjoint analysis. Em virtude dos objetivos, optou-se por aplicar dois níveis de análise: agregada e de subgrupos. A primeira é definida pelo agrupamento dos resultados, isto é, pela estimativa de uma função geral de utilidade. Já, a segunda caracteriza-se pela formação de segmentos com grupos de entrevistados que apresentam poder preditivo próximo aos modelos de nível individual (RAO, 2014). Especificamente, optou-se pela utilização da análise de cluster, caracterizada pelo desenvolvimento de subgrupos com base nas similaridades, isto é, na homogeneidade de perfis. Neste estudo, o critério de seleção dos grupos foram as preferências quanto aos grupos de referência (respostas do ranqueamento dos cartões). Em relação ao procedimento de agrupamento, utilizou-se o método de Ward e optou-se por uma solução com dois agrupamentos. Esclarecidos os procedimentos de coleta e análise dos dados, segue-se para a apresentação e a discussão dos resultados.

\section{RESULTADOS E ANÁLISES}

Nesta seção apresentam-se e analisam-se os resultados das duas etapas do estudo. Primeiramente, são analisados os achados da fase qualitativa. Na sequência, são discutidos os resultados da etapa quantitativa.

\subsection{RESULTADOS E ANÁLISES DA ETAPA QUALITATIVA}

A análise dos resultados da fase qualitativa estrutura-se em duas categorias definidas $a$ priori: cosméticos de alto envolvimento e influência dos grupos de referência. 


\subsubsection{Cosméticos de alto envolvimento}

$\mathrm{Na}$ literatura sobre o processo de decisão de compra, destaca-se que quanto mais alto o grau de envolvimento, maior o interesse e a relevância percebida (HOONSOPON; PURIWAT, 2016). Nesse sentido, embora as consumidoras entrevistadas mencionem a utilização de diferentes tipos e marcas de cosméticos, observa-se a predominância de duas categorias: batom e creme hidratante. $\mathrm{O}$ uso desses itens é unânime entre as respondentes. Contudo, salienta-se que os batons despertam maior interesse nas entrevistadas, enquanto os cremes parecem estar mais associados a uma necessidade - hidratar a pele: “[...] o preferido seria o batom" (E3); "Eu gosto muito de batom e creme pro rosto. Até o creme nem é tanto o gostar, é uma necessidade já que a pele fica muito ressecada" (E2). Além disso, a influência de outras pessoas no consumo de cremes parece ser pouco expressiva, seja pelo costume de escolher o que melhor se adapta ao tipo de pele da consumidora, seja pelo medo de indicação, similar a medicações.

Destaca-se também que apesar da crise econômica do país durante a aplicação da pesquisa, as entrevistadas afirmam não ter diminuído a compra de cosméticos: “[...] a gente tá sempre comprando [...] Nunca fica sem comprar" (E12); "Não deixo de comprar [maquiagem] [...." (E2). Esse comportamento reflete o fenômeno conhecido como "efeito batom" que indica o crescimento nas vendas de cosméticos durante recessões econômicas (CRUZ, 2016). Tendo em vista o nome utilizado para descrever o fenômeno, identifica-se o batom como um produto capaz de representar a categoria de cosméticos e, assim, delimitar o contexto da fase quantitativa deste estudo.

\subsubsection{INFLUÊNCIA DOS GRUPOS DE REFERÊNCIA}

A tomada de decisão de compra pode ser considerada um processo social devido à influência de outras pessoas na maneira com que um indivíduo pensa e sente em relação a um bem ou marca (AcKERMAN; MACINNIS; FOLKES, 2000; RILEY; GOSLINE, 2011).Nesse sentido, observa-se que os grupos próximos são os que exercem maior influência no processo de decisão de compra das entrevistadas. Especificamente, a família é evidenciada como o principal grupo de referência: "Eu converso bastante com as minhas irmãs, elas gostam também de maquiagem [...] E a minha mãe também" (E1); "As filhas gostam de se 
meter, de dar opinião" (E10). Essa identificação vai ao encontro dos estudos de Barbosa, Hor-meyll e Motta (2009) e Vasconcelos, Gomes e Leocádio (2015).

Nessa linha, percebe-se a presença de outros grupos próximos. Em consonância com a literatura, as amigas são identificadas pelas respondentes como um importante grupo de influência: "A gente tá sempre conversando com as amigas [...] tudo querendo se arrumar" (E8). O grupo referente as vizinhas também aparece nas falas de algumas das entrevistadas. No entanto, esse grupo não é tão relevante para essas consumidoras quanto as amigas: "Não, não. Elas são o grupo das vizinhas" (E3). Esse resultado difere do estudo de Vasconcelos, Gomes e Leocádio (2015), no qual os vizinhos representavam um grupo tão importante quanto à família. Essa divergência possivelmente relaciona-se ao contexto observado pelos autores, marcado por distinção social e status. Como o discurso dessas consumidoras sugere diferenças entre referências próximas, para incluí-las como fatores da análise conjunta optouse por dividi-las da seguinte forma: (1) familiares; (2) amigas; (3) vizinhas/colegas.

Além disso, observa-se na literatura a importância dos especialistas como grupos de referência (LEAL; HOR-MEYLL; PESSÔA, 2014). No que diz respeito especificamente ao comportamento de compra de batons de consumidoras de baixa renda, identifica-se que os únicos experts que as influenciam são as consultoras de venda: "A gente troca ideias [com a consultora], 'mistura esse com esse que fica legal' ou 'vai com essa marca que fica legal"' (E3); "Elas [revendedoras] ajudam a escolher o que fica melhor pra pele, 'ah aquele ali fica melhor pra ti"' (E11). Acredita-se que isso acontece porque, em geral, as consumidoras já conhecem as revendedoras. Isso faz com que as consultoras de venda deixem de ser apenas especialistas e passem a representar também um grupo de associação para essas consumidoras, pois há proximidade, afinidade cultural e confiança entre as entrevistadas e as revendedoras. Por essa razão, esse grupo de referência também foi incluído como um fator na análise conjunta do estudo quantitativo.

Além de especialistas, identifica-se que as celebridades são figuras idealizadas e, por isso, formam um relevante grupo de aspiração (MILLER; LACZNIAK, 2011; SCHIFFMAN; KANUK, 2000). Ao analisar as entrevistas com as consumidoras de baixa renda, constatou-se que nove (9) das doze (12) mulheres quando questionadas sobre a influência de celebridades alegaram que não costumam observar os produtos utilizados ou anunciados por personalidades famosas. Porém, no discurso das demais entrevistadas percebe-se maior preocupação em observar e, em algumas situações, seguir o comportamento das mulheres famosas, por exemplo: "Procuro mais os esmaltes que elas usam. [...] Ludmilla, tem uns esmaltes lindos, tem umas maquiagens perfeitas" (E3); "Atrizes da Rede Globo e as 
americanas. Beyoncé, Cameron Diaz, são as de maior influência na moda [...] O que elas tão usando normalmente é tendência. [...] eu vejo mais ou menos o que elas tão usando, aí vou no catálogo..." (E7). Diante disso, entende-se que essas entrevistadas inspiram-se e admiram essas celebridades.

A presença de influenciadores digitais mostra-se de maneira semelhante. Embora algumas entrevistadas tenham acesso à internet, apenas três (3) respondentes afirmam buscar informações sobre cosméticos em blogs. Dessas somente duas (2) afirmaram consultar a página de uma blogueira específica para aprender a fazer maquiagem e observar os produtos da moda: "Eu gosto muito da Alice Salazar, porque ela ensina fazer maquiagem, [...] só que realmente os produtos que ela fala é muito caro. Então a gente substitui" (E8); "Tem a Alice Salazar. [...] eu costumo olhar bastante" (E12). Embora os resultados observados indiquem que as celebridades e as blogueiras não representam grupos de referência expressivos para as consumidoras de baixa renda, optou-se por incluí-los como fatores da análise conjunta, pois algumas entrevistadas destacaram a sua presença em seu processo de decisão de compra.

Por fim, observa-se a influência de indivíduos com melhores condições financeiras, por exemplo, patroas e alguns parentes (BARROS, 2006; LIVRAMENTO; HOR-MEYLL; PESSÔA, 2013; VASCONCELOS; GOMES; LEOCÁDIO, 2015). Quando questionadas sobre a participação dessas referências em suas decisões de compra de cosméticos, as entrevistadas comentaram que não há uma influência direta, pois os produtos utilizados por esse grupo costumam ser mais caros e não condizem com a sua realidade: "[...] tu vê que fica bonito na pessoa, pergunta, né, 'ah, de que marca é'. Dependendo, tu já vê que tu não tem condições de comprar" (E10). Além disso, a opinião e o reconhecimento desse grupo de referência parecem ser importantes para essas consumidoras, por exemplo: "Se uma patroa disser que tá bonito o batom, eu vou me encher [de orgulho], com certeza!" (E7). Acredita-se que isso acontece porque as mulheres de baixa renda desejam ser reconhecidas socialmente e, mais do que isso, querem ser respeitadas por indivíduos de classes sociais hierarquicamente superiores (LIVRAMENTO, HOR-MEYLL, PESSÔA, 2013). Tendo em vista que receber um elogio ou uma sugestão desse grupo de referência têm um valor especial para essas consumidoras, optou-se por incluí-lo como fator na etapa quantitativa do estudo.

Os resultados dessa etapa são relevantes, pois possibilitam melhor compreensão sobre o comportamento das mulheres de baixa renda no que se refere ao consumo de cosméticos. Especificamente, esses achados oferecem um direcionamento adequado à segunda etapa do estudo, descrita na sequência. 


\subsection{RESULTADOS E ANÁLISES DA ETAPA QUANTITATIVA}

Nesta seção apresentam-se a caracterização do perfil da amostra, os resultados da Análise Conjunta em nível agregado e de cluster e a discussão desses resultados.

\subsubsection{Caracterização da amostra}

A amostra da etapa quantitativa incluiu a resposta de 53 mulheres de baixa renda de três grupos etários: menos de 25 anos $(33,96 \%)$, entre 25 e 50 anos $(33,96 \%)$ e mais de 50 anos (32,08\%). Identifica-se, ainda, que 43,4\% das respondentes são solteiras, 35,8\% são casadas, 41,5\% não possuem filhos e 39,6\% têm um ou dois filhos. Quanto à renda familiar mensal, observa-se que 34\% da amostra recebem até $\mathrm{R} \$ 1.320,00$, enquanto 35,8\% ganham entre $\mathrm{R} \$ 1.321,00$ e $\mathrm{R} \$ 1.760,00$ e 30,2\% recebem entre $\mathrm{R} \$ 1.761,00$ e $\mathrm{R} \$ 2.300,00$.

Em relação aos hábitos de compra e de consumo de batom salienta-se que a maioria das respondentes costuma adquirir esse tipo de cosmético com consultoras de venda (67,9\%). Outros canais são lojas especializadas em produtos de beleza (11,3\%), farmácia $(11,3 \%)$ e internet $(7,5 \%)$. Além disso, destacam-se os principais motivos para as respondentes utilizarem batom: sentir-se bem consigo mesma $(39,6 \%)$, hidratar e proteger os lábios $(39,6 \%)$ e sentir-se mais bonita $(15,1 \%)$.

\subsubsection{Análise conjunta agregada}

A análise de nível agregado caracteriza-se pelo agrupamento dos resultados de todos os respondentes (RAO, 2014). Assim, apresentam-se as utilidades parciais de cada nível dos fatores que permite mapear as preferências das entrevistadas (Tabela 1). Este modelo demonstra adequação e boa capacidade preditiva, dado que o coeficiente de correlação de Kendall's tau para os valores estimados e observados foi de 0,674 (sig=0,004).

$\mathrm{Na}$ Tabela 1 observa-se que a participação (sim) de familiares, amigas e consultoras de venda expressa utilidade de valor positivo, demonstrando boa aceitação por parte das respondentes. Por outro lado, a participação ( $\operatorname{sim}$ ) de vizinhas/colegas, celebridades, mulheres com melhores condições financeiras e blogueiras apresenta utilidade de valor negativo, sugerindo baixa aceitação. 
Tabela 1 - Utilidades estimadas - nível agregado

\begin{tabular}{l|c|c}
\hline \multirow{2}{*}{ FATOR } & NÍVEL & UTILIDADE ESTIMADA \\
\hline \multirow{2}{*}{ Familiares } & Sim &, 297 \\
\cline { 2 - 3 } Consultoras de venda & Não &,- 297 \\
\hline \multirow{2}{*}{ Amigas } & Sim &, 259 \\
\cline { 2 - 3 } & Não &,- 259 \\
\hline \multirow{2}{*}{ Vizinhas/colegas } & Sim &, 151 \\
\cline { 2 - 3 } Celebridades & Não &,- 151 \\
\cline { 2 - 3 } & Sim &,- 114 \\
\hline \multirow{2}{*}{ Blogueiras } & Não &, 114 \\
\cline { 2 - 3 } $\begin{array}{l}\text { Mulheres com melhores condições } \\
\text { financeiras }\end{array}$ & Sim &,- 161 \\
\cline { 2 - 3 } & Não &, 161 \\
\cline { 2 - 3 } & Sim &,- 176 \\
\hline
\end{tabular}

Fonte: elaborado pelos autores.

Para auxiliar o entendimento dessas preferências apresenta-se um gráfico com as utilidades parciais estimadas dos fatores para o nível "sim" (Gráfico 1).

Gráfico 1 - Utilidades estimadas - nível agregado

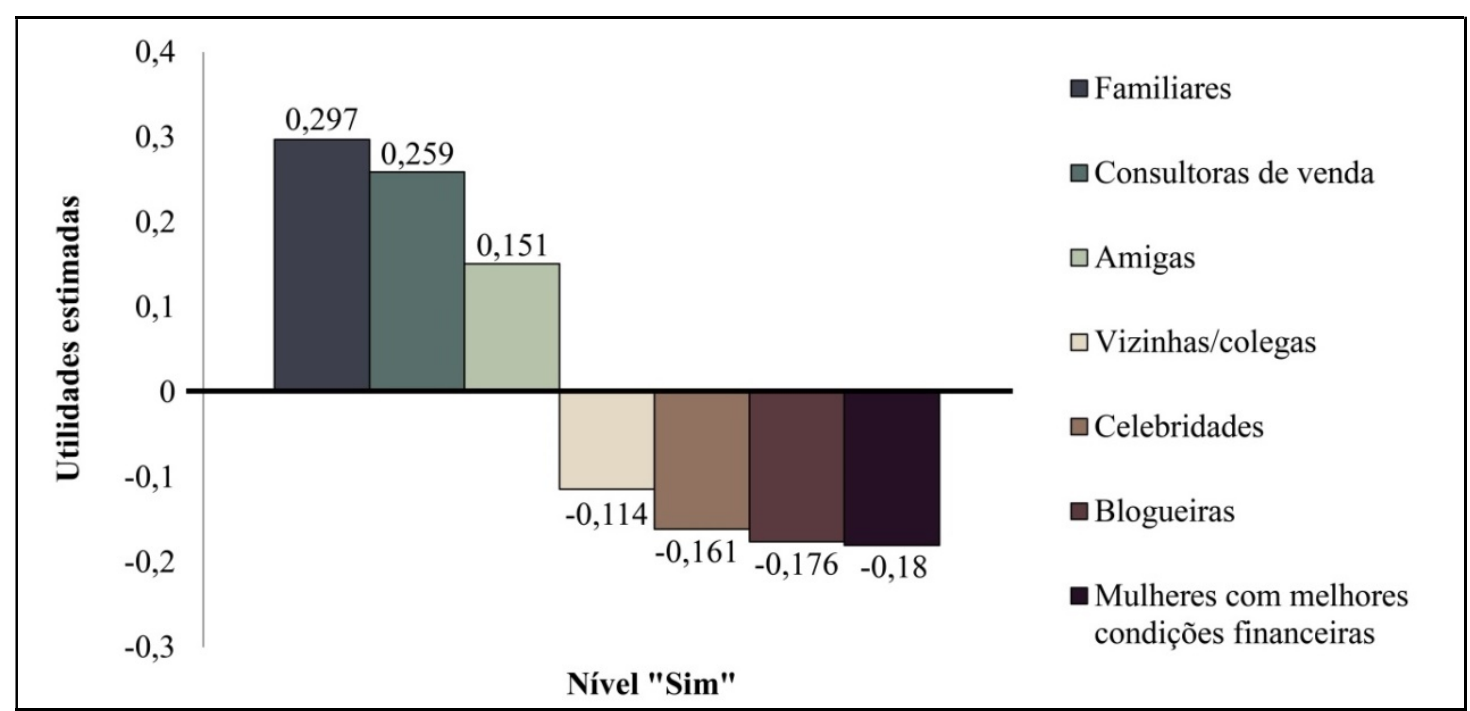

Fonte: elaborado pelos autores.

A análise agregada mostra-se útil para descrever os dados como um todo. No entanto, assume-se que os indivíduos são homogêneos em relação às funções de utilidade, de modo que o poder preditivo dessa demonstração costuma ser considerado baixo (RAO, 2014). Para 
manter a heterogeneidade dos respondentes, frequentemente se observam as funções de utilidade para segmentos. Assim, apresentam-se os resultados de conjoint analysis fundamentada em análise de aglomerados (clusters).

\subsubsection{Análise conjunta de clusters}

Inicialmente, realizou-se uma análise de cluster com o intuito de identificar diferentes agrupamentos, com base nas preferências das respondentes quanto aos grupos de referência que participam de seu processo de decisão de compra. Para isso, utilizou-se uma solução com dois grupos definida por meio do método de Ward. O primeiro cluster é composto por 39 casos (73,6\% da amostra), enquanto o segundo possui 14 casos (26,4\%). Esses segmentos foram identificados, respectivamente, como "Consumidoras pés-no-chão" e "Fãs de tendências".

$\mathrm{Na}$ sequência, realizou-se a conjoint analysis para cada segmento. As utilidades estimadas de cada grupo e cada nível de fator permitem mapear as preferências das respondentes e identificar diferenças entre os agrupamentos (Tabela 2). Ressalta-se que essas diferenças são estatisticamente significantes, pois a definição dos grupos obedeceu ao critério de homogeneidade característico da análise de cluster. Além disso, destaca-se que o teste Kendall's tau para o cluster $1(\tau=0,733$; sig=0,003) e para o cluster $2(\tau=0,796$; sig $=0,001)$ indica haver alta correlação entre as preferências observadas e estimadas. Em suma, o modelo demonstra adequação e boa capacidade preditiva.

Tabela 2 - Utilidades estimadas - clusters

\begin{tabular}{|c|c|c|c|}
\hline FATOR & NÍVEL & $\begin{array}{c}\text { Cluster 1 } \\
\text { "Consumidoras } \\
\text { pés-no-chão" }\end{array}$ & $\begin{array}{c}\text { Cluster 2 } \\
\text { "Fãs de } \\
\text { tendências" }\end{array}$ \\
\hline \multirow[t]{2}{*}{ Familiares } & Sim & ,285 & ,332 \\
\hline & Não &,- 285 &,- 332 \\
\hline \multirow{2}{*}{ Consultoras de vendas } & Sim & 285, & 189, \\
\hline & Não &,- 285 &,- 189 \\
\hline \multirow{2}{*}{ Amigas } & Sim & ,176 & ,082 \\
\hline & Não &,- 176 &,- 082 \\
\hline \multirow{2}{*}{ Vizinhas/colegas } & Sim &,- 140 &,- 042 \\
\hline & Não & , 140 & ,042 \\
\hline \multirow{2}{*}{ Blogueiras } & Sim &,- 211 &,- 077 \\
\hline & Não & 211 & 077 \\
\hline \multirow{2}{*}{$\begin{array}{l}\text { Mulheres com melhor condição } \\
\text { financeira }\end{array}$} & Sim &,- 294 & ,137 \\
\hline & Não & ,294 &,- 137 \\
\hline \multirow{2}{*}{ Celebridades } & Sim &,- 384 & ,458 \\
\hline & Não & ,384 &,- 458 \\
\hline
\end{tabular}

Fonte: elaborado pelos autores. 
Na Tabela 2 identifica-se que a participação (sim) de familiares, amigas e consultoras de venda expressa utilidade de valor positivo em ambos os grupos, demonstrando que as respondentes dos dois clusters valorizam a presença desses grupos de referência em seu processo de decisão de compra. Por outro lado, a participação de vizinhas/colegas e blogueiras apresenta utilidade de valor negativo nos dois grupos, sugerindo pouca aceitação das respondentes. Observa-se ainda que a presença $(\operatorname{sim})$ de celebridades e mulheres com melhores condições financeiras expressa valor negativo para o cluster 1 e valor positivo para o cluster 2. Esse resultado sugere que apenas as consumidoras "Fãs de tendências" valorizam esses grupos de referência. Para facilitar a visualização dessas preferências apresentam-se gráficos com as utilidades parciais estimadas dos fatores para o nível "sim" referentes ao cluster 1 (Gráfico 2) e ao cluster 2 (Gráfico 3).

\section{Gráfico 2 - Utilidades estimadas - cluster 1 (Consumidoras pés-no-chão)}

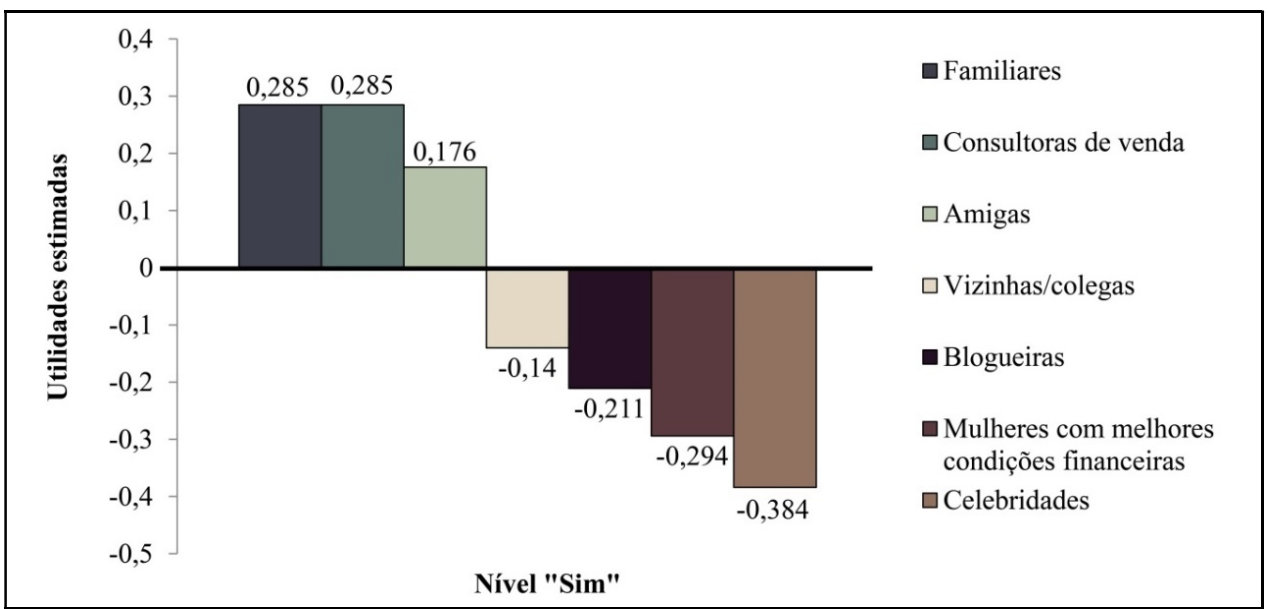

Fonte: elaborado pelos autores.

Gráfico 3- Utilidades estimadas - cluster 2 (Fãs de tendências)

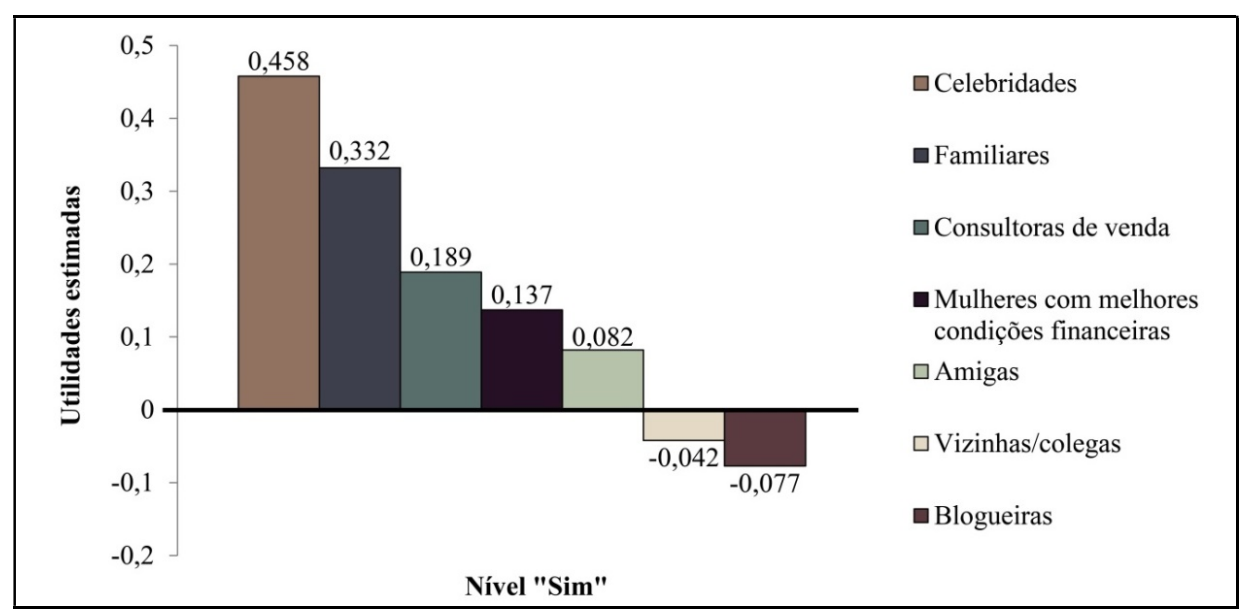

Fonte: elaborado pelos autores. 
Sabendo-se que quanto maior a utilidade, maior a preferência (HAIR Jr. et al., 2015), evidencia-se, no Gráfico 2, que os grupos mais valorizados pelas "Consumidoras pés-nochão" são os familiares e as consultoras de venda. Já na Figura 3, observa-se a preferência das "Fãs de tendências" pela presença, principalmente, das celebridades e das familiares. Além disso, ressalta-se que a baixa aceitação desse grupo em relação às vizinhas/colegas e às blogueiras é quase nula.

É importante salientar que as respostas referentes ao perfil das entrevistadas não demonstraram relação estatisticamente significantes com os clusters. Em outras palavras, é possível caracterizá-los a partir de suas preferências em relação aos grupos de referência, mas não com base em variáveis demográficas e de hábitos de compra e uso do produto. Para finalizar a seção de resultados e análises, articulam-se os resultados da etapa quantitativa, os achados da fase qualitativa e as informações referentes à literatura de marketing.

\section{DISCUSSÃO DOS RESULTADOS}

Com base nos resultados da etapa quantitativa, percebe-se que, ao abordar a amostra como um todo, algumas nuances relevantes são encobertas. Isso acontece porque a conjoint analysis agregada revela apenas uma fração das preferências das respondentes, enquanto a análise baseada em clusters apresenta uma perspectiva mais completa que permite identificar dois grupos com respostas distintas no que diz respeito à valorização dos grupos de referência. Assim, observa-se que as respostas das "Consumidoras pés-no-chão", refletem as preferências identificadas na conjoint analysis agregada, enquanto as "Fãs de tendências" demonstram especificidades quanto a isso.

Considerando-se os grupos de referência valorizados pelos clusters, destaca-se que ambos apreciam a participação de familiares, consultoras de vendas e amigas em sua tomada de decisão. Assim, compreende-se que as entrevistadas demonstram boa aceitação quanto à presença de indivíduos socialmente próximos e grupos de afinidade em sua decisão de compra (CHILDERS; RAO, 1992; ESCALAS; BETTMAN, 2003; SCHIFFMAN; KANUK, 2000). Esses resultados mostram-se alinhados com a literatura existente. Especificamente em relação aos familiares, sustentam-se os achados de Barbosa, Hor-meyll e Motta (2009) e de Vasconcelos, Gomes e Leocádio (2015) sobre a importância desse grupo no comportamento de consumidores de baixa renda. Quanto às consultoras de venda, entende-se que essa apreciação relaciona-se, sobretudo, à percepção das revendedoras como especialistas e à valorização que esse segmento atribui a vendedores que entendam a sua origem 
(PRAHALAD, 2005). Como as consumidoras criam vínculo e relacionamento com as consultoras, considera-se esse um grupo de afinidade para as mulheres de baixa renda.

$\mathrm{Na}$ sequência, observam-se os grupos de referência que não são valorizados pelos clusters identificados. Embora os vizinhos se destaquem na literatura como um importante grupo de contato, juntamente com os familiares, (BARROS, 2006; VASCONCELOS; GOMES; LEOCÁDIO, 2015), os resultados da conjoint analysis sugerem o contrário. Acredita-se que essa divergência relaciona-se às situações de compra abordadas. Enquanto o estudo de Vasconcelos, Gomes e Leocádio (2015) refere-se a móveis e eletrodomésticos, isto é, bens associados à ascensão e à distinção social (CASTILHOS; ROSSI, 2009), o presente estudo envolve uma categoria não relacionada a status (LIVRAMENTO; HOR-MEYLL; PESSÔA, 2013). As preferências quanto às blogueiras apresentam-se de maneira semelhante. Ainda que na etapa qualitativa, algumas entrevistadas tenham afirmado usar a internet e blogs para buscar informações sobre cosméticos, os resultados da segunda etapa sugerem que esse não é um grupo de referência valorizado pelas respondentes. Possivelmente a baixa aceitação das blogueiras relaciona-se às desconfianças do segmento de baixa renda quanto à internet (DIAS; HEMAIS, 2015).

Além disso, abordam-se as especificidades referentes às preferências das "Fãs de tendências". Mesmo que esse não seja o comportamento da maioria das respondentes, observa-se que esse segmento demonstra alta aceitação também em relação à presença de celebridades e mulheres com melhores condições financeiras. Entende-se, portanto, que, além dos grupos de afinidade, essas mulheres valorizam referências socialmente distantes (CHILDERS; RAO, 1992) e priorizam a influência de grupos de aspiração (ESCALAS; BETTMAN, 2003; SCHIFFMAN; KANUK, 2000). Especificamente em relação à valorização de mulheres com melhores condições financeiras, percebe-se que esse resultado está em conformidade com o estudo de Barros (2006), que evidencia a admiração das empregadas domésticas pelo estilo de vida das patroas.

Embora as diferenças entre esses agrupamentos não sejam estatisticamente relacionadas às variáveis de perfil - características demográficas e padrões de compra e uso do produto - os resultados sugerem que as entrevistadas demonstram orientações e motivações distintas que justificam suas preferências quanto aos diferentes grupos de referência. Por um lado, entende-se que as "Fãs de tendências" admiram e almejam o estilo de vida de determinados grupos de referência, ao mesmo tempo em que desejam obter reconhecimento social e respeito (LIVRAMENTO, HOR-MEYLL, PESSÔA, 2013). Em contrapartida, ao observar a não valorização desses dois grupos de referência pelas 
"Consumidoras pés-no-chão", entende-se que essas respondentes são altamente conscientes de suas limitações orçamentárias (LIVRAMENTO; HOR-MEYLL; PESSÔA, 2013), de modo que preferem observar e fundamentar sua decisão em sugestões de pessoas próximas, pertencentes à mesma realidade.

\section{CONSIDERAÇÕES FINAIS}

Os resultados encontrados cumprem com os objetivos propostos ao indicar que as mulheres de baixa renda valorizam diversos influenciadores em seu processo de compra. Diante disso, entende-se que a tomada de decisão dessas consumidoras se caracteriza como um processo social. Acredita-se que isso seja um reflexo do ambiente em que elas estão inseridas, o qual pode ser considerado coletivista e de alta distância do poder, devido à rede de sociabilidade e reciprocidade estabelecida, ao senso de comunidade formado e à forte hierarquização da sociedade brasileira (BARROS, 2006). Em contextos descritos dessa maneira, observa-se que as pessoas costumam formar suas opiniões e escolhas, principalmente com base em contatos pessoais e recomendações de terceiros (GOODRICH; DE MOOIJ, 2014).

Especificamente, os dados coletados permitem identificar dois tipos de consumidoras de baixa renda com preferências distintas em relação aos grupos de referência que participam de seu processo de decisão de compra. Nesse sentido, entende-se que a maioria das respondentes, isto é, as "Consumidoras pés-no-chão", são mulheres orientadas por seu ambiente imediato e por suas restrições financeiras, de modo que priorizam a racionalidade associada à compra e, por isso, recorram a grupos de afinidade - familiares, consultoras de venda e amigas - para tirar dúvidas e avaliar o custo-benefício de determinados produtos ou marcas. Em contrapartida, o grupo identificado como "Fãs de tendências", além de demonstrar preferência pelos grupos de contato, também valoriza importantes grupos de aspiração - celebridades e mulheres com melhores condições financeiras. Essa preferência, possivelmente, relaciona-se ao desejo de pertencer à sociedade de consumo e de acompanhar as novidades e tendências do mercado. Ainda que tenham limitações financeiras, elas tentam se adaptar a um estilo de vida almejado, incorporando produtos e marcas semelhantes que sigam as tendências do momento.

Os resultados provenientes da identificação dos clusters revelam a diversidade existente dentro do segmento de baixa renda, pois as consumidoras pertencentes a essa faixa de renda possuem preferências e comportamentos distintos entre si. Argumenta-se, portanto, 
que esse público não deve ser abordado e tratado como um grupo homogêneo de consumidores. A partir do exposto destaca-se que o presente estudo proporciona uma visão abrangente sobre o processo de decisão de compra de mulheres de baixa renda e evidencia a necessidade de se identificar e analisar as nuances presentes nesse segmento.

Como contribuição acadêmica deste estudo, destaca-se o método utilizado. Embora a aplicação mais frequente de Análise Conjunta seja referente ao desenvolvimento de novas ofertas (HAIR Jr. et al., 2005), evidencia-se que esta técnica pode ser usada em outras situações que envolvam a preferência das respondentes. Quanto às implicações práticas, sugere-se às empresas de cosméticos a utilização do apelo de consultoras de venda para atrair mulheres de baixa renda, devido ao reconhecimento e identificação por parte do público. Além disso, entende-se que o uso de celebridades pode ser uma boa estratégia, pois atingirá um grupo mais sensível às tendências e que, provavelmente, difundirá os cosméticos dentro do segmento.

Para finalizar, identificam-se limitações e sugestões para futuras pesquisas. Primeiramente, observa-se que o consumo de cosméticos não reflete a busca por distinção social e status, uma importante característica referida na literatura sobre o segmento de baixa renda (BARROS, 2006; CASTILHOS; ROSSI, 2009). Como existem diferentes modelos de tomada de decisão para diferentes produtos (MATTOS, 2007), propõe-se que futuros estudos analisem as preferências quanto aos grupos de referência em situações de compra associadas à distinção social e a status. Além disso, destaca-se uma limitação relacionada à caracterização dos clusters. Embora tenham sido identificadas diferenças nas preferências quanto aos grupos de referência, não foi possível descrever detalhadamente esses agrupamentos. Diante disso, sugere-se que futuros estudos busquem caracterizar o perfil dos grupos com base em variáveis psicográficas, por exemplo.

\section{REFERÊNCIAS}

ALMEIDA, M.I.S. et al. Quem lidera sua opinião? Influência dos formadores de opinião digitais no engajamento. RAC, v.22, n.1, art.6, p.115-137, jan/fev. 2018.

ASHMAN, R.; SOLOMON, M.R.; WOLNY, J. An old model for a new age: Consumer decision making in participatory digital culture. Journal of Customer Behaviour, v. 14, n. 2, p. 127-146, 2015. 
BARBOSA, P. HOR-MEYLL; L.F.; MOTTA, P.C. Uso de celular pré-pago por consumidores de baixa renda. In: ROCHA, Angela da; SILVA, J.F. (Org.). Consumo na base da pirâmide. Rio de Janeiro: Mauad X, 2009. p.113-132.

BARDIN, L. Análise de Conteúdo. 6. ed. São Paulo: Edições 70. 2011.

BARROS, C. Consumo, hierarquia e mediação: um estudo antropológico no universo das empregadas domésticas. In: XXX EnANPAD, 2006, Salvador. Anais... Rio de Janeiro: ANPAD, 2006.

BEARDEN, W.O.; ETZEL, M.J. Reference group influence on product and brand purchase decisions. Journal of Consumer Research, p. 183-194, 1982.

BRAGAGLIA, A.P.; BASTOS, L.L.A. O valor de aprovação social no consumo e na publicidade: uma discussão à luz da ética. Fragmentos de Cultura, v.27, n.2, p.258-270, abr./jun. 2017.

CASTILHOS, R.B.; ROSSI, C.A.V. Subindo o morro: consumo, posição social e distinção entre famílias de classes populares. In: ROCHA, Angela da; SILVA, J.F. (Org.). Consumo na base da pirâmide: estudos brasileiros. Rio de Janeiro: Mauad X, 2009, p.49-74.

CHAUVEL, M.A.; MATTOS, M.P.A.Z. Consumidores de baixa renda: uma revisão dos achados de estudos feitos no Brasil. Cadernos Ebape, v. 6, n. 2, p. 1-16, 2008.

CHILDERS, T.L.; RAO, A.R. The influence of familial and peer-based reference groups on consumer decisions. Journal of Consumer Research, v.19, p. 198-211, set.,1992.

CRUZ, F. Estratégias em tempos de incerteza: Aprendendo com consumidores de países emergentes em tempos de crise. Euromonitor International. 2016.

DIAS, J.P.S.; HEMAIS, M.W. Consumidores de baixa renda e compras on-line: receios em consumir pela internet. REGE-Revista de Gestão, v. 22, n. 1, p. 115-132, 2015.

ENGLIS, G.; SOLOMON, M.R. To be and not to be: lifestyle imagery, reference groups, and the clustering of America. Journal of Advertising, v. 24, n. 1, p. 13-28, 1995.

ESCALAS, J.E.; BETTMAN, J.R. You are what they eat: The influence of reference groups on consumers' connections to brands. Journal of Consumer Psychology, v. 13, n. 3, p. 3393482003.

FUNDAÇÃO GETÚLIO VARGAS. Centro de Excelência em Varejo. Definição de Baixa

Renda. 2011. [online]. Disponível em:

http://cev.fgv.br/sites/cev.fgv.br/files/Programa\%20Baixa\%20Renda\%20-

\%20DEFINI\%C3\%87\%C3\%83O\%20DE\%20BAIXA\%20RENDA\%20-\%20FINALx.pdf.

Acesso em: ago. 2016. Acesso em: 15 mar. 2019

REAd | Porto Alegre - Vol. 25 - No 2 - Maio / Agosto 2019 - p. 240-266 
GASKELL, G. Entrevistas individuais e grupais. In: BAUER, M.; GASKELL, G. (orgs.). Pesquisa qualitativa com texto, imagem e som: um manual prático. Petrópolis: Vozes, 2002 .

GOODRICH, K.; DE MOOIJ, M. How 'social' are social media? A cross-cultural comparison of online and offline purchase decision influences. Journal of Marketing Communications, v. 20, n. 1-2, p. 103-116, 2014.

GUILLEN-ROYO, M. Reference group consumption and the subjective wellbeing of the poor in Peru. Journal of Economic Psychology, v. 32, n. 2, 2011, p. 259-272, 2011.

HAIR, Jr. J.F. et al. Análise multivariada de dados. 5. ed. Porto Alegre: Bookman, 2005. HOONSOPON, Danupol; PURIWAT, Wilert. The effect of reference groups on purchase intention: Evidence in distinct types of shoppers and product involvement. Australasian Marketing Journal (AMJ), v. 24, n. 2, p. 157-164, 2016.

LEAL, G.P.A.; HOR-MEYLL, L.F.; PESSÔA, L.A.G.P. Influence of virtual communities in purchasing decisions: The participants' perspective. Journal of Business Research, v. 67, n. 5, p. 882-890, 2014.

LIVRAMENTO, M.N.; HOR-MEYLL, L.F.; PESSÔA, L.A.G.P. Valores que motivam mulheres de baixa renda a comprar produtos de beleza. RAM. Revista de Administração Mackenzie, v. 14, n. 1, p. 44-74, 2013.

MATTOS, M.P.A.Z. O consumidor de baixa renda e sua relação com as marcas no processo de decisão de compra de refrigerante e sabão em pó. Rio de Janeiro: PUCRJ, 2007.

MEDEIROS, J.F.; CRUZ, C.M.L. Comportamento do consumidor: fatores que influenciam no processo de decisão de compra dos consumidores. Teoria e Evidência Econômica, Passo Fundo, v. 14, Ed. Especial, p. 167-190, 2006.

MILLER, F.M.; LACZNIAK, G.R. The Ethics of Celebrity-Athlete Endorsement. Journal of Advertising Research, v. 51, n. 3, p. 499-510, 2011.

MURADIAN, M.B.A. et al. Influência de Blogs Patrocinados no Comportamento da Consumidora de Cosméticos. In: XVII SemeAd, 2014. São Paulo. Anais... São Paulo: FEA, 2014.

NOGAMI, V.K.C.; PACAGNAN, M.N. Consumo da Base da Pirâmide: Uma análise bibliométrica do estado da arte na produção acadêmica no Brasil. In: XXXV EnANPAD, 2011, Rio de Janeiro. Anais... Rio de Janeiro: ANPAD, 2011.

PARK, C.W.; LESSIG, V.P. Students and housewives: Differences in susceptibility to reference group influence. Journal of Consumer Research, v.4, n. 2, p. 102-110, 1977. 
PORTO, R.B.; OKADA, S.I. Comportamento do consumidor em canais cruzados e seus benefícios: validação de escala para avaliar o desempenho do processo de compra. Revista Brasileira de Gestão de Negócios, v.20, n.2, p.443-460 2018.

PRADO, K.P.A. et al. A preferência da marca no processo de decisão de compra: um estudo exploratório no segmento de baixa renda. Organizações em Contexto, v. 10, n. 19, p. 357 $382,2014$.

PRAHALAD, C.K. A riqueza na base da pirâmide: como erradicar a pobreza com o lucro. Porto Alegre: Bookman, 2005.

PRAHALAD, C.K.; LIEBERTHAL, K. The end of corporate imperialism. Harvard Business Review, v.81, n.8, p. 109-117, ago. 2003.

RAO, V.R. Applied conjoint analysis. New York: Springer, 2014.

RILEY, B.; GOSLINE, R. The Dark Side of Social Groups: How Social Reference Groups Inhibit Consumption. Advances in Consumer Research, v. 39, p.150-154, 2011.

ROCHA, A.; SILVA, J.F. Inclusão social e marketing na base da pirâmide: uma agenda de pesquisa. RAE-eletrônica, v. 7, n. 2, 2008. Disponível em:

https://rae.fgv.br/sites/rae.fgv.br/files/artigos/10.1590_S1676-56482008000200007.pdf. Acesso em: 24 jan. 2019.

SCHIFFMAN, L.G.; KANUK, L.L. Comportamento do consumidor. Rio de Janeiro: LTC, 2000 .

UZUNOĞLU, E.; KIP, S.M. Brand communication through digital influencers: Leveraging blogger engagement. International Journal of Information Management, v. 34, n. 5, p. 592-602, 2014.

VASCONCELOS, A.I.T.; GOMES, D.M.O.A.; LEOCÁDIO, Á.L. “Tititi” na Baixa Renda: Explorando o Compartilhamento do Significado dos Bens. In: XXXIX EnANPAD, 2015, Belo Horizonte. Anais... Rio de Janeiro: ANPAD, 2015. 